\title{
On the Current State of Ground-based Transmission Spectroscopy of Planet Atmospheres
}

\author{
Lisa Nortmann ${ }^{1}$, Stefan Dreizler ${ }^{1}$ and Jacob Bean ${ }^{2}$ \\ ${ }^{1}$ Institut für Astrophysik, Universität Göttingen, \\ Friedrich-Hund-Platz 1, 37077 Göttingen, Germany \\ email: nortmann, dreizler@astro.physik.uni-goettingen.de \\ ${ }^{2}$ The Dep. of Astronomy \& Astrophysics, University of Chicago, \\ 5640 S. Ellis Ave, Chicago, IL 60637, United States \\ email: jbean@oddjob.uchicago.edu
}

\begin{abstract}
In response to the large number of exoplanet detections, the characterization of these planets has become a major focus of exoplanet science. Transiting planets are of particular interest as they allow us to investigate the transmission of their atmospheres. Our group uses ground-based facilities like the ESO/VLT to probe the atmosphere of hot Jupiters with the technique of spectrophotometry. In our preliminary results for the hot Jupiters WASP-17b and WASP-31b we find the reachable precision to be limited by instrumental systematic noise rather than photon noise. We discuss the source of the noise and suggest two approaches to correct it.
\end{abstract}

Keywords. instrumentation: spectrographs, occultations

\section{Introduction}

Over 200 of the approximately 980 exoplanets detected in the last two decades are known to transit in front of their host star in our line of sight. Transiting exoplanets provide us with the unique opportunity to examine the transmission spectrum of their atmosphere. This is due to the fact that during transit a small fraction of the observed star light passes through the upper atmosphere of the exoplanet. With the precision reachable by using the current generation of telescopes and instruments, measuring this faint signal is feasible for planets which exhibit highly extended atmospheres. In order to help design the instruments that will be able to reach the precision needed to probe the atmosphere of rocky and potentially habitable exoplanets, it is imperative to employ the existing methods to the planets for which they are currently applicable. This allows us to identify and study the factors that currently limit the precision of the measurements and, thus, help us to develop new observing methods and improve the existing ones.

\section{Method and Observations}

In order to probe the exoplanet atmosphere, we utilize the fact that the planet will appear larger in wavelength regions in which its atmosphere's constituents exhibit absorption than it will in other wavelength regions. As the planet radius can be obtained 

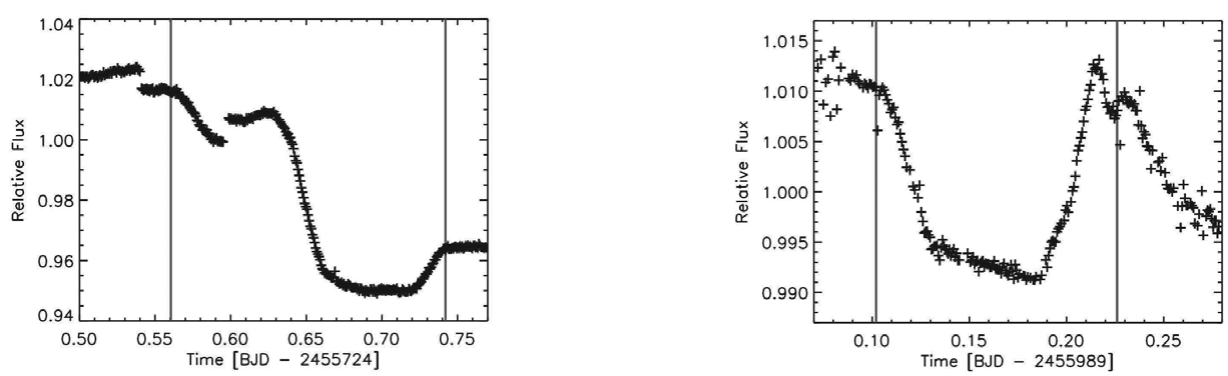

Figure 1. Example light curves of WASP-17b (left) and WASP-31b (right) showing the distortion. The grey lines mark in- and egress of the transit.

from transit observations, a transmission spectrum can be inferred if the wavelength dependent planet radius measurements are obtained from transit observations in several narrow band passes.

We observed the two hot Jupiter planets WASP-17b and WASP-31b during a whole transit event in June 2011 and in March 2012, respectively. The observations were conducted from the ESO/VLT facility using the FORS2 instrument in multi-object spectroscopy mode. In both cases, approximately 300 spectra were taken for the planet host star and a reference target. From these spectra, we extracted transit light curves at several $20 \mathrm{~nm}$ wide wavelength channels. We found these light curves to be strongly distorted by non-astrophysical systematic noise (Fig. 1), which could be traced back to the longitudinal atmospheric dispersion corrector (LADC) of the telescope. The LADC consists of two prisms, whose distance can be modulated to optimally correct the atmospheric dispersion. These prisms exhibit a spatial and wavelength dependent transmittance variability. Due to the Earth's rotation during the observations, the star light passed through the LADC at different spatial positions causing the measured intensity to vary. During the observations of WASP-17b the prism distance was additionally changed twice, causing the jumps that can be seen in the light curve.

\section{Analysis}

$W A S P-17 b$ : For WASP-17b we corrected the systematic noise by modeling it as a function of the LADC prism distance $\Delta_{L A D C}$ and the Parallactic Angle $\theta_{P A}$. For the determination of the wavelength dependent planet radius, i.e. the transmission spectrum, the data was fitted with a model of the following form:

$$
M(\lambda)=T\left(r_{p}(\lambda)\right)\left(a_{1}(\lambda) \cdot \Delta_{L A D C}\right)\left(a_{2}(\lambda) \cdot \theta_{P A}+a_{3}(\lambda) \cdot \theta_{P A}{ }^{2}+a_{4}(\lambda) \cdot \theta_{P A^{3}}\right)
$$

Where $T\left(r_{p}(\lambda)\right)$ is an analytical transit model (Mandel \& Agol, 2002) and $r_{p}(\lambda), a_{1,4}(\lambda)$ are the free fit parameters for each wavelength channel, respectively.

WASP-31b: For WASP-31b we used the approach recently suggested by Waldmann (2012), which is based on the assumption that the systematic noise can be described as a linear combination of several noise signals, all contributing to the signal measured in each wavelength channel data with different weights. If this is the case, then blind source separation algorithms can be used to de-mix the input data into the individual signal components. We divided each wavelength channel by the white light curve to dispose of any wavelength independent noise components and then used the MULTI-COMBI algorithm (Tichavsky et al. 2008) to identify the signals comprising the residual data. 

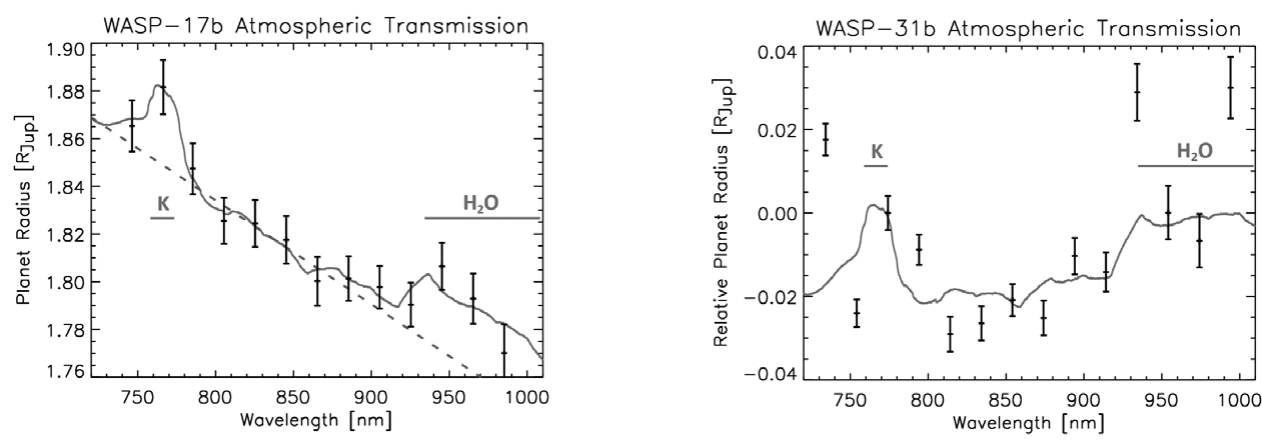

Figure 2. The preliminary results for the transmission spectrum of WASP-17b (left) and WASP-31b (right) plotted together with a theoretical model (solid line). In the case of WASP-17b, the model is tilted by an additional linear polynomial function (dotted line) to fit the data. Typical errors are of the order of $\approx 0.01 R_{\mathrm{Jup}}$ and $\approx 0.005 \mathrm{R}_{\mathrm{Jup}}$ for WASP-17b and WASP-31b, respectively.

We found two significant signal components $s i g_{1}$ and $s i g_{2}$. The residual data was then fitted with a model of the form:

$$
M(\lambda)=T\left(r_{p}(\lambda)\right) \exp \left(a_{1}(\lambda) \cdot \operatorname{sig}_{1}+a_{2}(\lambda) \cdot \operatorname{sig}_{2}\right) / T\left(r_{p}\left(\lambda_{0}\right)\right)
$$

Where $r_{p}\left(\lambda_{0}\right)$ is the planet radius given by Anderson et al. $(2011)$ and $r_{p}(\lambda), a_{1,2}(\lambda)$ are the free fit parameters for each wavelength channel, respectively.

\section{Results}

The preliminary results for both planets are shown in figure 2 together with a theoretical model calculated by T. Barman as described in Barman (2007) (private communication) including potassium and water absorption.

The transmission spectrum of WASP-17b exhibits a strong trend towards larger radii with lower wavelength. This might be caused by Rayleigh scattering in the planet atmosphere or the existence of unocculted star spots on the host star. We can, however, not fully exclude that the trend is caused by residual systematic noise, insufficiently corrected by our model approach. The theoretical model shown in figure 2 (left) was multiplied with a linear polynomial function of wavelength (indicated by the dotted line) to compensate for this wavelength trend.

\section{Preliminary Conclusion}

Instrumental systematic noise is frequently found in spectrophotometric transit data. Several approaches to its correction have been proposed by us and other authors. While these corrections allow us to derive results for exoplanet transmission spectra which exhibit very small statistical errors, possible residual systematic errors might still be underestimated.

\section{References}

Anderson, D. R., Collier Cameron, A., Hellier, C., Lendl, M., Lister, T. A., Maxted, P. F. L., Queloz, D., Smalley, B., Smith, A. M. S., Triaud, A. H. M. J., West, R. G., Brown, D. J. 
A., Gillon, M., Pepe, F., Pollacco, D., Ségransan, D., Street, R. A., \& Udry, S. 2011, A\&̋A, 531,60

Barman, T. 2007, ApJ, 661, L191-L194

Mandel, K. \& Agol, E. 2002, ApJ, 580, L171

Tichavsky, P., Koldovsky, Z., Yeredor, A., Gomez-Herrero, G., \& Doron, E. 2008, IEEE Tr. Neural Networks, 19,3, 421-430

Waldmann, I. P. 2012, ApJ, 747, 12 